\title{
IDEA: TRANSFORMACIONES DE UN MODELO PARA LA GESTIÓN TECNOPEDAGÓGICA
}

\section{IDEA: TRANSFORMATIONS OF A MODEL FOR TECHNO-PEDAGOGICAL MANAGEMENT}

Elvia Garduño Teliz

Docente investigadora de la UAGro. Actualmente doctorando en pedagogía en la Universidad Nacional Autónoma de México. Ponente y conferenciante en congresos nacionales e internacionales sobre tecnologías en la educación. Formadora de profesores. Cuenta con estándares nacionales e internacionales de competencia en la impartición de cursos presenciales, diseño de cursos de formación en línea y evaluación de competencias.

Principal línea de investigación: procesos tecnopedagógicos; innovación y educación.

Correo electrónico: [elvia_garduno_teliz@hotmail.com].

\section{RESUMEN}

El empleo de las Tecnologías de la Información y la Comunicación (TIC) en los procesos formativos, requiere la integración de tecnología, pedagogía y didáctica en procesos recursivos de aprendizaje y empoderamiento. Para atender a esta necesidad se plantea la gestión tecnopedagógica para la formación en la virtualidad y en la presencialidad. 
El artículo tiene por objetivo presentar la evolución recursiva de un modelo de gestión tecnopedagógica denominado Integracciones Dimensionales de Empoderamiento y Aprendizaje (IDEA).

El lector conocerá las diferentes versiones del modelo, sus procesos de construcción, deconstrucción y reconstrucción, así como sus elementos, dimensiones y fases para transitar del diseño instruccional hacia los procesos tecnopedagógicos. La metodología aplicada en el modelo es la investigación acción, que lo hace proactivo por encima de lo prescriptivo. El proceso de la investigación acción se ha llevado a cabo a través de la aplicación formativa del modelo. En consecuencia, IDEA ha sido implementado en la gestión de Objetos Digitales de Aprendizaje (ODA) y Cursos Masivos Abiertos en Línea, en los que se han tenido experiencias favorables por parte de los docentes en cuanto a su adaptabilidad tecnopedagógica como modelo de uso. La prospectiva del modelo se orienta hacia los estudiantes, por lo que se plantean posibles nichos estratégicos para favorecer el empoderamiento y el aprendizaje.

Palabras clave: modelo educacional, gestión, TIC.

\section{ABSTRACT}

The use of Information and Communication Technologies (ICT) in the formative processes, requires the integration of technology, pedagogy and didactics in recursive processes of learning and empowerment. For this reason, it is proposed the techno-pedagogical management for training in virtuality and in face-to-face. The article aims to present the recursive evolution of a techno-pedagogical management model called Dimensional Integrations of Empowerment and Learning (IDEA).

The reader will know the different versions of the model, its construction, deconstruction and reconstruction processes, as well as its elements, dimensions and phases to move from instructional design to techno-pedagogical processes. The methodology applied in the model is action research, which makes it proactive rather than prescriptive. The process of action research has been carried out through the formative application of the model. As a result, IDEA has been implemented in 
the management of Digital Learning Objects (ODA) and Open Massive Online Courses (MOOC), in which teachers have had favorable experiences in terms of their technical and pedagogical adaptability as a model of use. The prospect of the model is oriented towards the students, so that possible strategic niches are proposed to favor the empowerment and the learning.

Key words: educational model, management, ICT.

\section{INTRODUCCIÓN}

Al aplicar las TIC a los procesos formativos, existe el reto de integrar la pedagogía y la didáctica. En palabras de Thakur (2015, p. 182): «cada maestro debe saber cómo usar la tecnología, la pedagogía y el contenido de la materia de manera efectiva en su enseñanza diaria del aula. Está claro que la mera introducción de la tecnología al proceso educativo no es suficiente». En este sentido, aparecen los enfoques tecnopedagógicos a través de los cuales las TIC se transforman en Tecnologías de Aprendizaje y Conocimiento (TAC), así como en Tecnologías de Empoderamiento y Participación (TEP), tanto en contextos formales como informales.

Un marco tecnopedagógico lo representa el modelo Tecno Pedagogical Content Knowledge (TPACK) que determina que cualquier propuesta formativa debe fundamentarse en conocimientos tecnológicos, pedagógicos y de contenido. El modelo TPACK queda limitado a la inclusión de la didáctica y la gestión. En consecuencia, su implementación como referente tecnopedagógico es complicada para un docente o estudiante que transita de las TIC a las TAC y TEP, en procesos formativos formales y en contextos tanto virtuales como presenciales.

Para contar con un modelo de uso simple y adaptativo se presenta la evolución recursiva de un modelo de gestión tecnopedagógica denominado Integracciones Dimensionales de Empoderamiento y Aprendizaje (IDEA), deconstruido y construido a través de un proceso de investigación acción participativa. La gestión es el centro del modelo IDEA y está representada por actividades tecnopedagógicas realizadas 
por los usuarios del modelo - docentes o estudiantes- para transitar en las dimensiones de la información, el conocimiento y el aprendizaje. También representa la oportunidad para integrar las fases y los elementos del modelo.

Las fases propuestas son: diseño, producción, implementación didáctica, seguimiento y evaluación. Los elementos son los ambientes, el contenido y dos términos que representan la transición de roles de los usuarios del modelo a través de la gestión: webcente y participantes integractivos.

Primero se muestran los conceptos y la terminología de base de IDEA, lo mismo que sus relaciones e integracciones. Después se presenta la evolución del modelo como parte del ciclo investigación acción. Finalmente se identifican propuestas formativas para la aplicación del modelo IDEA en estudiantes.

\section{CONCEPTOS Y TERMINOLOGÍA DE BASE DEL MODELO IDEA}

En el campo de la educación, los modelos han sido recurridos ampliamente para presentar propuestas y resultados de investigación educativa. Un modelo didáctico es una «construcción teórico-formal que basada en supuestos científicos e ideológicos pretende interpretar la realidad escolar y dirigirla hacia determinados fines educativos» Regla Alicia Sierra Salcedo (como se cita en De Armas, N., Valle, 2011, p. 17).

Un modelo es el paso de la teoría a la práctica, de la reflexión a la acción. Para integrar la tecnopedagogía en la construcción del modelo, se incluye como referente el Modelo de conocimiento de contenido tecnopedagógico - (Pedagogical Technological Content Knowledge o TPCK) por Mishra y Koehler (2006, p. 1025) — que presenta un marco dirigido a los docentes que contiene una superposición de cuatro elementos originados con el uso de las TIC: tecnológico, pedagógico, contenido y conocimiento. 
En palabras de los autores del modelo: «el hecho de saber cómo usar la tecnología no es lo mismo que saber cómo enseñar con ella» (Mishra y Koehler, 2006, p. 1033). Lo tecnopedagógico guarda relación con el término «literacidad digital académica». Reynolds y Hanes (2009) la definen como el conjunto de actividades básicas que todo ser humano adquiere para aprender a lo largo de su vida en contextos virtuales para crear, administrar, publicar, socializar, colaborar, investigar, navegar y jugar con los contenidos, entidades y espacios digitales con fines formativos.

A diferencia del TPACK, IDEA abarca además de la tecnología y la pedagogía, la gestión y la didáctica. El modelo IDEA, es una construcción y deconstrucción teórico-metodológica, resultado de la investigación educativa para el empoderamiento de los usuarios, «en el desarrollo de su autonomía a partir de su sentido colaborativo e inteligencia colectiva» (Levy, 2004).

Las bases pedagógicas del modelo IDEA son el constructivismo social, la teoría sociocultural, la teoría de las inteligencias múltiples y el conectivismo.

El constructivismo social, desde la perspectiva de Schunk (2012, p. 231), establece que «las construcciones de una persona son verdaderas para ella, pero no necesariamente para los demás».

La teoría sociocultural de Vygotsky (2009, p. 75) relaciona a IDEA como un artefacto para el desarrollo de las funciones psíquicas superiores «como procesos mediatizados...».

La teoría de las inteligencias múltiples en la perspectiva de Gardner (2010, p. 117) refiere a la inteligencia como «una capacidad que se centra en unos contenidos específicos».

La visión conectivista de Siemens (2005, p. 21) acerca del conocimiento, se caracteriza por dejar de lado las «jerarquías y contenedores estáticos. La organización actual consiste en redes dinámicas y ecologías-modelos capaces de adaptación (ajustar y reaccionar a los cambios)». 


\subsection{Elementos del modelo}

Se replantean los elementos del triángulo pedagógico que originalmente se centraban en el estudiante, docente y contenidos. Desde el enfoque sociocultural, se retoma la teoría de la actividad de Leontiev y se destaca la relación entre prácticas y actividad en la que se «acentúa una forma de relación dialéctica entre el sujeto y el objeto» (Montealegre, 2005, p. 34). De esta manera se consideran las pasiones, los intereses y las necesidades de los participantes, se relacionan contextualmente con los contenidos para darles significatividad y se concretan a partir de una transformación práctica en un ambiente de aprendizaje, presencial, virtual o mixto. Los elementos del modelo son:

La gestión tecnopedagógica es el centro del modelo; es un conjunto de actividades de convergencia que se incrustan en todo el modelo, orientadas al logro de una intención formativa. Se relaciona con las siguientes dimensiones:

La gestión de la información se enfoca a la alfabetización informacional, para la calidad en su uso ante el riesgo latente de la infoxicación por cantidad excesiva, cambios y periodicidad.

La gestión del conocimiento se refiere a las actividades en que los participantes generan, contrastan, comparten, construyen y difunden el saber científico, como insumo para el aprendizaje.

La gestión del aprendizaje implica la concreción y significatividad en la práctica escolar y cotidiana para el cambio personal. El aprendizaje se representa por la significación, la movilización y la transferencia de habilidades, y conecta la información con el conocimiento a través de los contenidos digitales. Las actividades son didácticas o adidácticas y están orientadas por teorías pedagógicas.

La gestión administrativa está encaminada a las políticas educativas sobre el uso de TIC en los sistemas educativos formales. Estas políticas detectan y/o atienden la problemática de la infraestructura para el acceso, la conectividad, alfabetización, aplicación y concreción de las TIC en los procesos formativos. 
Webcente. Es el profesional de la docencia que comprende en sus concepciones teóricas y en la concreción de su práctica a la pedagogía, la tecnología y la didáctica como elementos transversales para la mediación de contenidos en ambientes de aprendizaje. Es un agente de cambio en la formación de personas, adopta y transforma sus roles, visualiza a la virtualidad y la presencialidad como espacios y oportunidades de aprendizaje para ir de las TIC a las TAC y TEP.

Participante integractivo. Desde una perspectiva tecnopedagógica se refiere a los estudiantes que integran y accionan el modelo IDEA para la gestión de su aprendizaje de manera colaborativa y autogestiva, permitiéndoles lograr la literacidad digital académica en el ámbito de las TIC, TAC y TEP.

Ambientes. Componentes pedagógicos, didácticos, psicológicos, sociales y tecnológicos orientados hacia el logro de una intención formativa. Los docentes y estudiantes interactúan en la virtualidad y presencialidad, asumiendo roles diferentes. Por ejemplo, ambos pueden ser diseñadores, productores, evaluadores y mediadores. En consecuencia, los ambientes permiten la transición de roles del docente al webcente y del estudiante a participantes integractivos.

Contenido. Conjuntos de conocimientos para el aprendizaje. Los contenidos deben estar didácticamente organizados y estructurados para la realización de la intención formativa. Se integran de manera intrínseca y extrínseca, porque la razón y la acción se conectan en actividades presenciales y virtuales.

\subsection{Dimensiones del modelo IDEA}

La gestión del modelo transita en tres dimensiones, representadas por la sociedades de la información, el conocimiento y el aprendizaje.

La sociedad de la información conceptualizada por Masuda, Y. (1980) se decanta por el acceso, la generación, distribución y el manejo significativo del conjunto de datos organizado y sistematizado, conocido como información. En el contexto de este trabajo, se relaciona 
directamente con la virtualidad en la perspectiva de ser un derecho inherente a la condición del ser humano.

La sociedad del conocimiento es un concepto propuesto por Peter Drucker surgido a partir de las transformaciones sociales. Desde la perspectiva de Krüger (2006, p. 5), el conocimiento es un factor de cambio social, a saber:

El concepto actual de la sociedad del conocimiento no está centrado en el progreso tecnológico, sino que lo considera como un factor de cambio social entre otros, como por ejemplo, la expansión de la educación. Según este enfoque, el conocimiento será cada vez más la base de los procesos sociales en diversos ámbitos funcionales de las sociedades. Crece la importancia del conocimiento como recurso económico, lo que conlleva a la necesidad de aprender a lo largo de la vida. Pero igualmente crece la conciencia del no-saber y la conciencia de los riesgos de la sociedad moderna.

Dentro de la utopía del capitalismo moderno, la sociedad del conocimiento se relaciona con la economía del conocimiento en cuyo concepto se privilegia al saber por encima del trabajo, para generar riqueza y valor. En este sentido, se destaca la labor de los docentes puesto que «ellos deben tomar lugar entre la sociedad de intelectuales más respetada, moverse más allá del salón de clases para ser y preparar a sus estudiantes como ciudadanos del mundo» (Hargreaves, 2002, p. 3).

La sociedad del aprendizaje es un concepto emergente, en palabras de Su (2010, p. 8): «es la respuesta a cambiar el mundo». El valor del aprendizaje es tanto intrínseco como extrínseco, puesto que quien lo posee y está dispuesto a aprender, adquiere una visión distinta y más amplia de los mundos en los que coexiste - entre la presencialidad y la virtualidad-, mientras que la sociedad que tiene una apertura hacia el aprendizaje, se beneficiará cuando el individuo integre comunidades de aprendizaje en las que comparta y amplíe el suyo propio. De este modo, la sociedad del aprendizaje se concentra en procesos socioculturales, en experiencias individuales y colaborativas que se internalizan pero, a la vez, se externalizan en un proceso recursivo en el que la persona amplía su perspectiva y 
mejora su prospectiva como agente de cambio en los contextos tanto presenciales como virtuales de los que forma parte, esto va más allá del ámbito económico y se encamina hacia la literacidad digital.

Las relaciones entre la información, el conocimiento y el aprendizaje constituyen procesos permanentes de construcción y deconstrucción formativa a través de la gestión con el modelo IDEA.

\subsection{Fases del modelo}

Para concretar el modelo se aplican sus fases de manera recursiva y no jerárquica.

Diseño. Es una fase de planeación en la cual se vislumbran elementos de interés para el participante integractivo y/o el webcente. La interactividad cognitiva concatena recursos y actividades en función de los niveles cognitivos establecidos en la intención pedagógica y la situación didáctica (entendida como una intención del webcente para concretar en el entorno un conflicto o necesidad que requiera una movilización de conocimientos, habilidades y actitudes para el aprendizaje), o una situación adidáctica planteada por el participante integractivo. El diseño debe atender a la pertinencia y a la contextualización, tomando como punto de partida además de la intención formativa, las pasiones, los intereses, las inteligencias y los estilos de aprendizaje. En un sentido clave responde a la pregunta ¿qué propuesta educativa digital se realizará?

Producción. Es una fase de ejecución para concretar el diseño dirigido a la audiencia. La elección del software es necesaria y depende del diseño, así como de la infraestructura y los requerimientos para implementarlo. De esta manera, se amplían las posibilidades de convergencia de ambientes de aprendizaje mixtos. En un sentido clave responde a la pregunta ¿con qué herramientas digitales va a hacerse?

Implementación. Es la concreción práctica en ambientes de aprendizaje semiformales e informales, la cual puede supeditarse a una planeación y organización didáctica que contenga la estrategia para la 
aplicación significativa del modelo. Responde a la pregunta ¿cómo se aplicará?

Seguimiento y evaluación. Es una fase transversal en todo el proceso. Consiste en la valoración práctica y metacognitiva de los resultados del modelo. La validez del modelo se sustenta en la participación en los diferentes momentos y las formas de atención mediata e inmediata. La evaluación del modelo se conjuga con la evaluación del aprendizaje, si demuestra incidir en el logro de la intención formativa. Responde a la pregunta ¿qué puede mejorarse?

El modelo es cíclico, recursivo y complejo, por lo que no existe una jerarquía o fase de inicio, siendo esta característica uno de los elementos centrales de su adaptabilidad y flexibilidad en diferentes dimensiones, disciplinas y ámbitos.

\subsection{Relaciones e integracciones del modelo IDEA}

Las relaciones del modelo se concretan a través de la gestión. Un ejemplo de relación entre las dimensiones del modelo es el siguiente:

En primer lugar, se accede a la información a través dispositivos electrónicos para realizar una búsqueda intencionada que involucre procesos de identificación, selección y presentación. Estos procesos se realizan en Internet a través de sitios web, comunidades de práctica y/o aprendizaje, incluso de expertos conectados. En los contextos presenciales, se integran a las personas que pueden ser los docentes, profesionales, familiares y compañeros de estudio, además de los espacios públicos como bibliotecas, salas de lectura, museos, lugares arqueológicos, reservas naturales, zoológicos, espacios universitarios, cines, plazas, y todos aquellos espacios digitales y presenciales en los que los actores educativos puedan acceder a lo que buscan y en el momento en que lo requieren.

En segundo lugar, la información no solamente es presentada, sino que debe ser evaluada por el grupo escolar quien, con la mediación del docente, la valida y transfiere a la práctica en contextos y situaciones 
específicas por lo que, simultáneamente, se integran la información, el conocimiento y el aprendizaje. Esa transferencia se realiza a través de estrategias didácticas orientadas hacia la resolución de problemas, la investigación y la toma de decisiones; de tal manera, que se relacione lo que se sabe con lo nuevo y se resignifique.

En consecuencia, esta resignificación provocará en el estudiante el aprendizaje a partir de la internalización y externalización de lo que sabe, al transformar su conducta e incidir propositivamente en la interacción social. Desde esta perspectiva, el conocimiento, el aprendizaje y la información tienden hacia la comunidad: «el conocimiento informa al aprendizaje; lo que aprendemos informa a la comunidad; y la comunidad a su vez crea conocimiento» (Siemens, 2005, p. 15).

Las integracciones son acciones que integran las acciones de la gestión en las dimensiones:

\section{Cuadro 1. Integracciones del modelo IDEA}

\begin{tabular}{|l|l|l|}
\hline Fases & \multicolumn{1}{|c|}{ Integracciones } & Dimensiones \\
\hline \multirow{3}{*}{ Diseño } & $\begin{array}{l}\text { Conocer los estilos, las pasiones, necesidades } \\
\text { y expectativas de aprendizaje. "Lo que me gus- } \\
\text { taría aprender y hacer» (Delval, 1996, p. 90). } \\
\text { Se establece la intención formativa, los nive- } \\
\text { les cognitivos y la mediación. "Lo que puedo } \\
\text { aprender y hacer» (Delval, 1996, p. 90). }\end{array}$ & $\begin{array}{l}\text { Gestión del } \\
\text { conocimiento. }\end{array}$ \\
\hline
\end{tabular}




\begin{tabular}{|c|c|c|}
\hline Fases & Acciones $\quad$ Integracciones & Dimensiones \\
\hline Producción & $\begin{array}{l}\text { Se elige para utilizar la herramienta web } \\
2.0 \\
\text { Se elige el espacio virtual para la publicación } \\
\text { o difusión. } \\
\text { Se aplica la herramienta con base en el diseño } \\
\text { realizado. } \\
\text { Se configura el espacio virtual para su publi- } \\
\text { cación, garantizando la accesibilidad, facili- } \\
\text { dad de navegación, consulta y/o descarga. }\end{array}$ & $\begin{array}{l}\text { Gestión de } \\
\text { la información. }\end{array}$ \\
\hline $\begin{array}{l}\text { Implementación } \\
\text { didáctica }\end{array}$ & $\begin{array}{l}\text { Se orientan las situaciones didácticas: de } \\
\text { formulación, acción, validación e institucio- } \\
\text { nalización (Brousseau, 1986). } \\
\text { Se establece la estructura didáctica: inicio, } \\
\text { desarrollo o cierre. } \\
\text { Se determina la estrategia didáctica. } \\
\text { Se concatenan las actividades con las } \\
\text { evidencias de aprendizaje. } \\
\text { Se aplica, explica y replica lo elaborado por } \\
\text { el modelo IDEA. }\end{array}$ & $\begin{array}{l}\text { Gestión del } \\
\text { aprendizaje. }\end{array}$ \\
\hline $\begin{array}{l}\text { Seguimiento } \\
\text { y evaluación }\end{array}$ & $\begin{array}{l}\text { Se diseñan y aplican } \\
\text { instrumentos de evaluación. } \\
\text { Se evalúa y realimenta el aprendizaje. } \\
\text { Se promueve la reflexión sobre la base de } \\
\text { la participación, para la mejora continua } \\
\text { del modeloy de las evidencias de su aplicación. }\end{array}$ & $\begin{array}{l}\text { Todas las } \\
\text { dimensiones. }\end{array}$ \\
\hline
\end{tabular}

Fuente: Elaboración propia, modificado de Garduño, E. (2016). 


\section{LA INVESTIGACIÓN ACCIÓN EN LA EVOLUCIÓN DEL MODELO IDEA}

Como referente del proceso de investigación acción se presenta la perspectiva adaptada de Zuber-Skerrit (2003, p. 1) quien establece un enfoque emancipador.

Inicialmente se buscó la colaboración crítica, autocrítica de los docentes, con el propósito de empoderarse a través de su participación activa. La investigación se orienta a que el docente reflexione e incida en las acciones formativas propias y las que realiza con los estudiantes. Desde la perspectiva de Lewin (1946, como se cita en Nocedo, I., et. al., 2015, p. 80), se presenta un espiral permanente de reflexión y acción fundamentada en la unidad entre la práctica y el proceso investigativo, y se desarrolla a partir de dos ideas cruciales: la decisión del grupo y el compromiso con la mejora.

La decisión del grupo, se relaciona con la negociación del acceso que se ha tenido, tanto en contextos virtuales como presenciales, para implementar el modelo IDEA. En la presencialidad se trabajó con docentes del nivel medio superior y superior de la UAGro. En la virtualidad se aplicó para la gestión de Cursos Masivos Abiertos en Línea (conocidos como MOOC por sus siglas en inglés), a través de los cuales también fue publicado y difundido.

El compromiso con la mejora se reflejó en el interés y motivación por participar en la investigación acción para conocer y aplicar el modelo IDEA, así como en las propuestas realizadas para deconstruir y reconstruir el modelo.

El proceso de la investigación comprendió las siguientes fases:

Fase planeación estratégica. Se identificaron variables, premisas y dimensiones para dar respuesta a la pregunta orientadora ¿cómo implementar el modelo de gestión IDEA en modalidad mixta? Se realizó un diagnóstico para la construcción de la primera versión del modelo. Se aplicó una encuesta a docentes sobre sus perspectivas en el uso de 
las TIC con enfoques formativos, además de una entrevista a expertos. Las respuestas se analizaron y triangularon para generar una estrategia de implementación presencial en forma de talleres:

\section{Cuadro 2. Organización de talleres}

\begin{tabular}{|l|l|}
\hline \multicolumn{1}{|c|}{ Taller } & \multicolumn{1}{|c|}{ Evidencias } \\
\hline Conferencia demostrativa del & 1. Invitación. \\
Modelo IDEA. & 2. Registro de asistencia. \\
\hline $\begin{array}{l}\text { Taller «Creatividad para la innovación } \\
\text { educativa». }\end{array}$ & $\begin{array}{l}\text { 3. Infografías. } \\
\text { 4. Cómic pedagógico. }\end{array}$ \\
\hline Taller ODA Interactivos. & 5. ODA Interactivos. \\
\hline Taller Estrategias tecnodidácticas. & $\begin{array}{l}\text { 6. Estrategia de trabajo para la } \\
\text { implementación didáctica de } \\
\text { los ODA. }\end{array}$ \\
\hline Taller Entornos de aprendizaje virtual. & $\begin{array}{l}\text { 7. Blogs educativos. } \\
\text { 8. Plataformas educativas } \\
\text { virtuales. }\end{array}$ \\
\hline $\begin{array}{l}\text { Curso-Taller elaboración de secuencias } \\
\text { didácticas. }\end{array}$ & $\begin{array}{l}\text { 9. Secuencia para la } \\
\text { implementación didáctica. }\end{array}$ \\
\hline Curso-Taller: evaluación. & $\begin{array}{l}\text { 10. Instrumentos de evaluación } \\
\text { del ODA. }\end{array}$ \\
\hline
\end{tabular}

Fuente: Elaboración propia.

En todos los talleres se trabaja con el modelo IDEA, por lo que se obtienen evidencias que los docentes se comprometieron a aplicar en sus grupos escolares. 
Fase aplicación de lo planeado. Se refiere a la puesta en marcha del plan, así como los efectos temporales en torno de su funcionamiento. En los talleres presenciales, los docentes y directivos sugirieron cambios en el orden, en función a sus expectativas y necesidades. Por lo tanto, el proyecto se flexibilizó. El tiempo fue una variable interviniente, puesto que requirieron más tiempo para la gestión por sus ocupaciones laborales.

Fase de observación, evaluación y autoevaluación. Se emplearon instrumentos de observación no participante, encuestas a estudiantes y cuestionarios reflexivos a docentes. Esta fase fue simultánea a la de aplicación, con miras a la mejora de la práctica docente, por lo que la observación, realimentación propositiva, participación activa y acción metacognitiva fueron aspectos importantes. Sin embargo, hubo negativas por parte de los docentes a las fases de implementación didáctica, evaluación y seguimiento, puesto que se negaron a ser observados en su práctica en el aula y, así, proporcionar evidencias al respecto. La realimentación propositiva se realizó mediante plenarias en las que compartieron sus ODA.

Fase reflexión crítica y autocrítica. La participación activa se constató a través de cuestionarios reflexivos. No obstante, esta fase se vio limitada por la experiencias en el uso tecnopedagógico de las TIC, aunado a que los docentes no estuvieron dispuestos a continuar en los talleres por el tiempo, su carga de trabajo y los procesos de certificación de sus planteles.

Fase toma de decisiones. Pese a la falta de continuidad con el proyecto, el modelo tuvo la oportunidad de ampliar su aplicación a través de los MOOC. En esta experiencia se realizó un curso denominado Gestión de Objetos Digitales de Aprendizaje, en dos ediciones: al respecto se incorporó el análisis cualitativo a través de la etnografía virtual. Esta fase hizo recursivo el proceso de la investigación, por lo que se orientaron las acciones hacia el contexto virtual en el que se implementó el modelo IDEA. La dualidad de su aplicación en la gestión del MOOC y en la gestión de ODA ha fortalecido las premisas, variables y los objetivos, así como replanteado nuevas líneas y metodologías de investigación.

De esta experiencia derivaron propuestas de los docentes que lo aplicaron, originando tres versiones del modelo. 


\section{VERSIONES DEL MODELO IDEA}

La primera versión, fue resultado del diagnóstico. Se representó a través de un diagrama de Venn para mostrar las dimensiones e integraciones entre las fases propuestas; las flechas representan un proceso cíclico y recursivo entre un triángulo de elementos propuestos inicialmente: participantes (docentes y estudiantes), contenidos y prácticas.

La primera versión del modelo se publicó con licencia internacional Creative Commons en versión 4.0 con reconocimiento a la autora sin fines comerciales y con obra derivada. Este licenciamiento se realizó como parte de la publicación de un blog en la dirección http:/ / webcente. blogspot.mx/ para compartir los materiales que se presentaban a los docentes en los talleres presenciales.

La primera versión, apareció en el año 2016, como parte de un capítulo del libro «El enfoque por competencias, gestión, innovación y prospectiva» (Garduño, 2016).

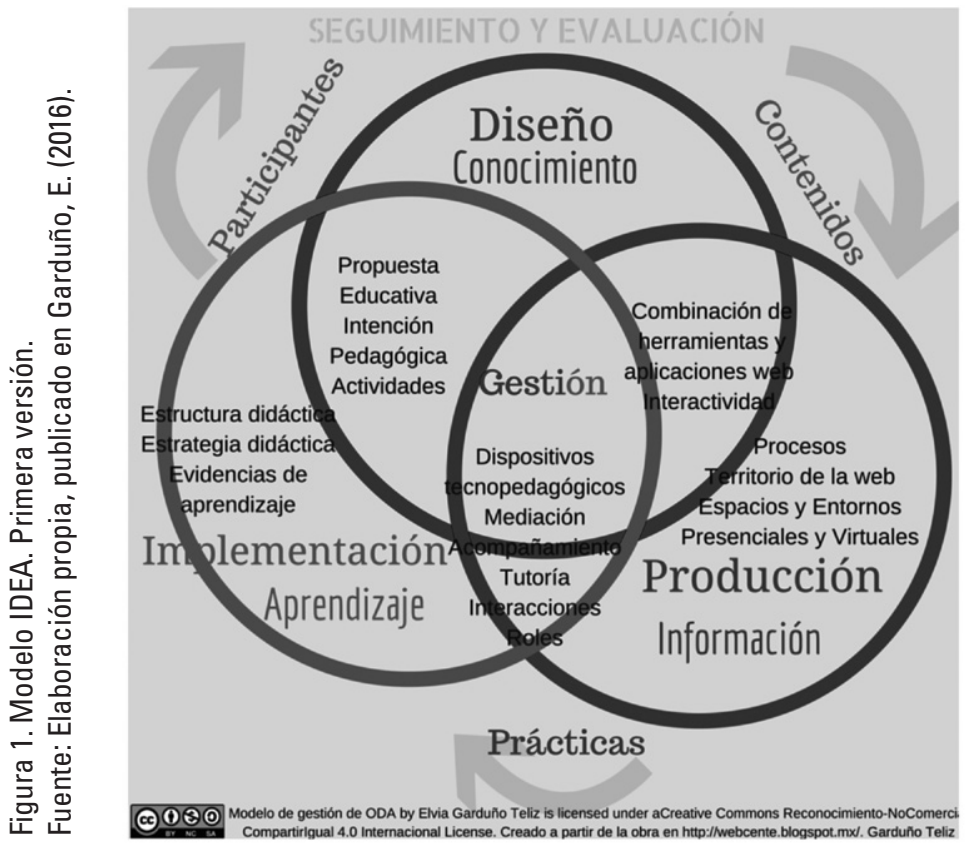


En los talleres se observó la necesidad de diferenciar los roles que asumen los usuarios del modelo. Por esta razón, en la segunda versión se sustituyeron los elementos iniciales del triángulo por un cuadrilátero de elementos tecnopedagógicos de base para realizar la gestión: webcente, contenidos, participantes integractivos y ambientes. La práctica como elemento del modelo anterior, se incrustó en un ambiente de aprendizaje, para ampliar las posibilidades de integracción. También se incorporó a la reflexión un elemento transversal dentro de las dimensiones del modelo. Otra novedad de esta versión es la inclusión de las situaciones didácticas dentro de la fase de implementación. La segunda versión se presenta en la siguiente figura:

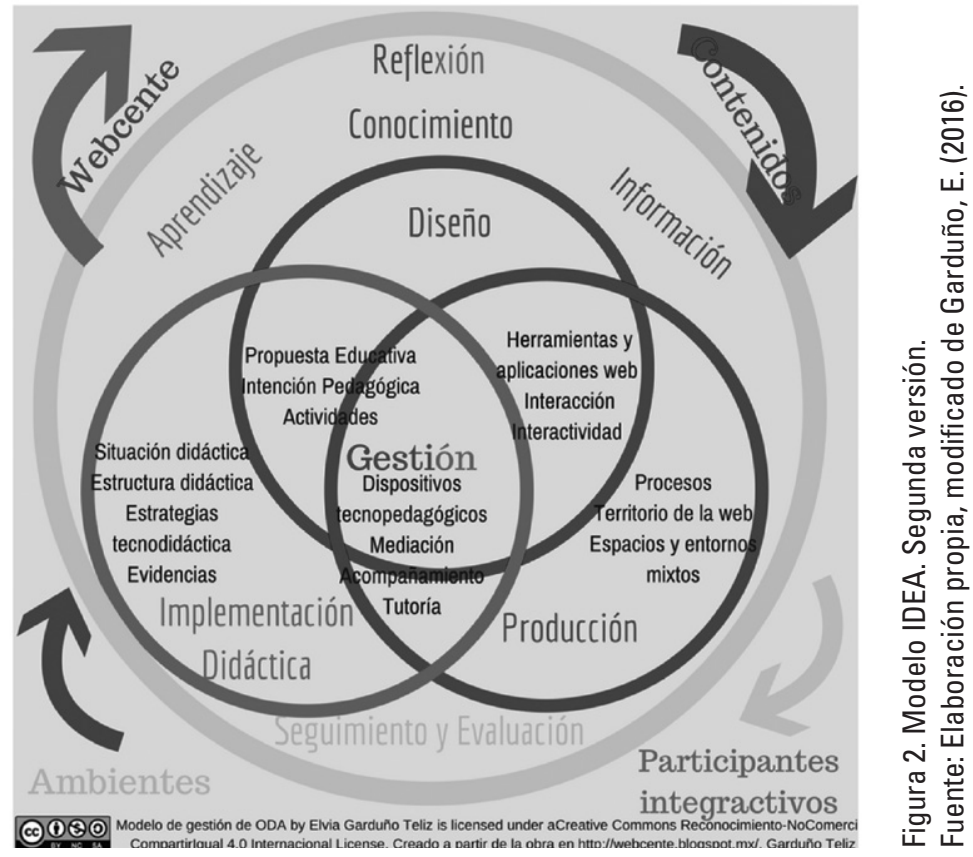

La segunda versión se aplicó en el MOOC Gestión de ODA. El MOOC está publicado dentro del sitio web ACADÉMICA — plataforma interactiva impulsada por Teléfonos de México (TELMEX)—, conocida también como comunidad digital de conocimiento. Este sitio reúne 
contenidos educativos de prestigiadas instituciones de Educación Superior y Centros de Investigación Internacionales, con quienes se trabaja para «compartir el conocimiento y hacerlo accesible a todo aquel que desea aprender y desarrollar su potencial» (Académica, 2017). El MOOC permitió difundir ampliamente la propuesta, capacitar a los docentes en la implementación del modelo, así como obtener perspectivas sobre su uso y propuestas de mejora.

La URL del MOOC en su segunda edición es http:/ / academica. $\mathrm{mx} /$ \#/ curso/695 y está sujeta a disponibilidad en función de la programación de la plataforma.

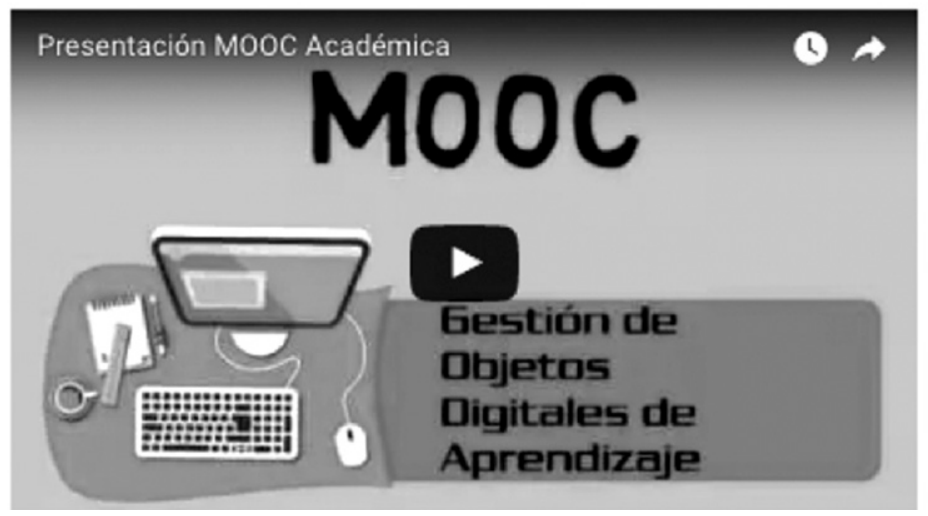

Figura 3. Interfaz del MOOC en la primera edición.

Fuente: http://academica.mx/\#/

Consultada el día 10 de octubre de 2016.

La metodología empleada fue la netnografía de cada edición del MOOC. De manera presencial la etnografía se asocia más con la interacción cara a cara; sin embargo, para Hine (2004, p. 13) «la etnografía consiste en que un investigador se sumerja en el mundo que estudia por un tiempo determinado y tome en cuenta las relaciones, actividades y significaciones que se forjan entre quienes participan en los procesos sociales de ese mundo». En este sentido, la virtualidad implica, para el investigador, un reto mayor para construir sentidos, significados e interpretaciones. 
Se emplea un «enfoque "autoetnográfico", lo que significa que estudié experiencias de los estudiantes en un curso que yo mismo enseñé» (Wasson, 2014, párr. 13). Esto concuerda con la concepción conectivista del aprendizaje como conocimiento para la acción, conexiones en las que nosotros mismos aportamos nuestro conocimiento y mejoramos su estado actual (Siemens, 2014, p. 5).

La primera edición del MOOC se realizó en el segundo semestre de 2016. El objetivo de la netnografía fue evaluar la aplicación modelo IDEA en modalidad mixta por los participantes del MOOC. Para lograrlo se realizó la observación de identificadores, el análisis de contenido, un cuestionario virtual, la revisión de la bitácora de registros así como una encuesta de satisfacción del MOOC.

La segunda edición del MOOC concluyó en el mes de abril de 2017, por lo que el proceso netnográfico está en curso.

De la triangulación del análisis etnográfico, realizado en la primera edición del MOOC, se derivaron corolarios que resaltan la importancia de cuatro competencias digitales como base para el uso tecnopedagógico del modelo de gestión: creatividad, gestión de información, colaboración y autogestión. En consecuencia, éstas se integraron a una tercera versión del modelo para aplicarlo en propuestas formativas alternas.

La tercera versión se emplea actualmente en la gestión de dos MOOC: uno denominado «competencias digitales» y otro «aprendizaje invertido». 


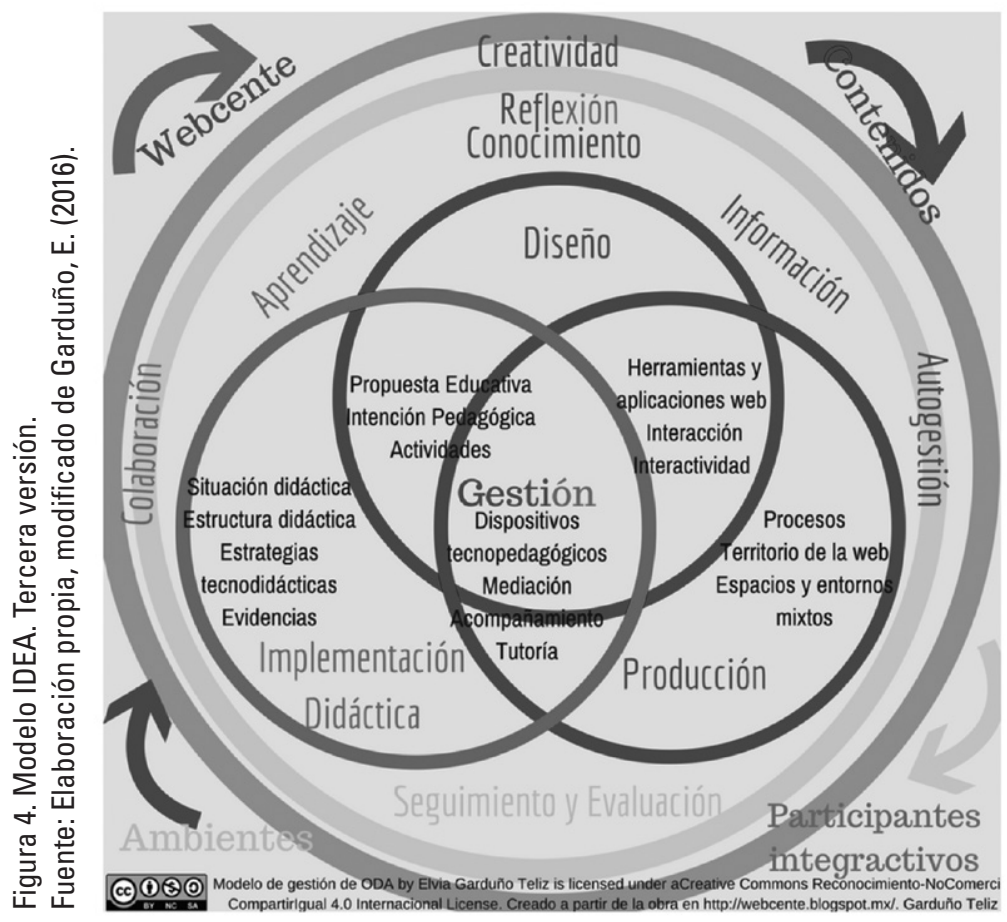

Esta versión se deconstruirá a partir de los resultados obtenidos del análisis netnográfico de los MOOC en los que fue implementado. Se espera generar una cuarta versión orientada hacia nuevas propuestas de aplicación del MOOC, con otros actores y escenarios educativos.

\section{PROPUESTAS DE APLICACIÓN}

Los estudiantes son los actores en los que el modelo IDEA plantea centrarse para generar nuevos procesos recursivos. Hasta ahora, los procesos tecnopedagógicos del modelo han tomado la voz de los docentes y generado progresos en aspectos pedagógicos y didácticos de la gestión. La experiencia buscada en los estudiantes ha replanteado las propuestas de aplicación de IDEA hacia las narrativas digitales, las rutas de aprendizaje, la ciudadanía digital y los Entornos Personales de 
Aprendizaje. Estas experiencias se plantean en los niveles educativos de educación secundaria, preparatoria, licenciatura y posgrado e incluyen a las TIC, TAC y TEP, como parte del proceso.

En las narrativas digitales, la gestión crea espacios de expresión digital en los que se generan procesos reflexivos de autoconocimiento y autogestión colaborativa.

En las rutas de aprendizaje, la gestión del qué, cómo, dónde y cuándo aprender, combinará la virtualidad y la presencialidad en el proceso. Una primera experiencia la constituye el MOOC Aprendizaje Invertido.

Los Entornos Personales de Aprendizaje se gestionarán para concretar rutas de aprendizaje, incluyendo redes y comunidades de aprendizaje.

En la ciudadanía digital, IDEA gestionará, sobre un perfil base, acciones para movilizar y transferir competencias digitales. Un acercamiento se realiza con el MOOC competencias digitales.

El elemento tecnopedagógico estará basado en los perfiles de literacidad digital. Aunque requieren un proceso de mediación, IDEA incidirá en habilidades que fortalezcan el aprendizaje a lo largo de la vida. Se retoma, así, la propuesta de Prensky (2011) para trabajar una pedagogía basada en las pasiones de los estudiantes y transferirlas a propuestas digitales de aprendizaje.

\section{CONCLUSIÓN}

El modelo IDEA ha mejorado notablemente con la investigación acción. Una de las razones de la factibilidad de su aplicación, es que los docentes no requieren formar parte de equipos especializados de materiales educativos, ya que pueden gestionar propuestas educativas de manera individual, colaborativa o colegiada. Los espacios de gestión consideran la modalidad mixta, lo que amplía las posibilidades de acción e integración. Adicionalmente, el manejo de herramientas, espacios y aplicaciones de la web 2.0 hacen más simple la producción. 
La inclusión de lo tecnopedagógico hace cíclico y recursivo el proceso, y fortalece los aspectos didácticos como, por ejemplo, la adaptabilidad de las herramientas web al diseño tecnopedagógico y no al revés.

Finalmente, la participación en la gestión como en la mejora del modelo, es un factor de empoderamiento dado su carácter propositivo y no prescriptivo.

Estas circunstancias han favorecido, en la práctica, la reconstrucción de las versiones del modelo IDEA.

Como toda experiencia formativa, el modelo puede continuar con su evolución recursiva. La labor tecnopedagógica no es solamente el empoderamiento y transición de roles de los actores educativos, sino también generar una literacidad digital académica, basada en la concepción de la virtualidad como parte de la realidad y en la convergencia de las TIC, las TAC y las TEP en la gestión de la información, el conocimiento y el aprendizaje. IDEA continuará su aplicación ahora hacia los estudiantes, a quienes se busca empoderar reconociendo su capacidad de crear su propia ruta de aprendizaje.

\section{REFERENCIAS}

Académica Comunidad Digital de Conocimiento (2015). Académica. México. Recuperado de http:/ / academica.mx/\#/

De Armas, N. y Valle, A. (2011). Resultados Científicos en la Investigación Educativa. (L. L. S. Peláez, Ed.) (1ª ed.). La Habana, Cuba: Editorial Pueblo y Educación.

Brousseau, G. (2006). Theory of didactical situations in mathematics: Didactique des mathématiques, 1970-1990 (Vol. 19). Springer Science \& Business Media.

Delval, J. (1996). Los fines de la educación. Siglo XXI de España Editores. 
Gardner, H. (2010). La inteligencia reformulada. Las inteligencias múltiples en el siglo XXI ( $1^{a}$ ed.). Barcelona: Paidós.

Garduño, E. (2016). Modelo IDEA: Modelo de uso para aplicar procesos tecnopedagógicos. En CIMTED (Ed.), El enfoque basado en competencias, gestión, innovación y prospectiva (CIMTED, p. 133). Medellín, Colombia.

Hargreaves, A. (2002). Teaching in the knowledge society. Technology Colleges Trust Vision 2020-Second International Online Conference, (December 2002), 1-14. http://doi. org/10.1007/978-1-4614-1083-6

Krüger, K. (2006). El concepto de «Sociedad del conocimiento». Biblio 3W. Revista Bibliográfica de Geografía y Ciencias Sociales, XI(683), 1-14. http:// doi.org/10.1007/s13398-014-0173-7.2

Levy, P. (2004). Inteligencia Colectiva. Por una antropología del ciberespacio. Recuperado de http://www.textos.pucp.edu.pe/ textos/descargar/2281.pdf

Mishra, P. \& Koehler, M. J. (2006). Technological pedagogical content knowledge: A framework for teacher knowledge. Teachers College Record. http:/ / doi.org/10.1111/j.1467-9620.2006.00684.x

Montealegre, R. (2005). La actividad humana en la psicología histórico-cultural. Avances en Psicología Latinoamericana, 23(1), 33-42. Recuperado de http:// www.redalyc.org/articulo. oa?id=79902304

Prensky, M. (2011). Enseñar a nativos digitales. Madrid: Ediciones SM.

Reynolds, R. (2016). Defining, designing for, and measuring «social constructivist digital literacy» development in learners: a proposed framework. Educational Technology Research and Development, 64(4), 735-762. http:/ / doi.org/10.1007/s11423-015-9423-4 
Schunk, D. (2012). Teorías del Aprendizaje. Una perspectiva educativa. (P. Educación, Ed.) (6 ed.). México.

Siemens, G. (2014). Conectivismo: Una teoría de aprendizaje para la era digital. Recuperado de www.diegoleal.org/docs/2007/ Siemens(2004)-Conectivismo.doc

Siemens, G. (2005). Knowing Knowledge. Anales de la Real Academia Nacional de Medicina, 122, http:/ / doi.org/10.2307/2102859

Su, Y. (2010). The learning society: Two justifications National Kaohsiung Hospitality College Taiwan. Australian Journal of Adult Learning, 50(1), 10-25.

Thakur, N. (2015). A Study on Implementation of Techno-Pedagogical Skills, Its Challenges and Role To Release At Higher, 182-186.

Wasson, C. (2014). «Desmitificando MOOCs: Un revelador Estudio Etnográfico de la Educación en Línea» [Publicación de Blog]. Blog Ethnography matters. Recuperado de:

http: / / ethnographymatters.net/es/blog/2014/02/18/ demystifying-moocs-an-eye-opening-ethnographic-study-ofonline-education/

Vygotsky, L. S. (2009). Pensamiento y Lenguaje. (S. A. de C. V. Ediciones Quinto Sol, Ed.) (11a reimpr). México.

Zuber-Skerritt, O. (2003). New directions in action research. (T. and F. e-Library, Ed.). London. Recuperado de:

https://goo.gl/xUKf6I 